\title{
Identification of factors determining anxiety, depression and aggression, with particular emphasis on pain in Polish adolescents and young adults with cystic fibrosis
}

\author{
GRAŻYNA CEPUCH ${ }^{1, A, ~ B, ~ D-G}$, LUCYNA TOMASZEK², A-D, F, G, LIDIA PAWLIK², A, B, F
}

${ }^{1}$ Nursing and Midwifery Institute, Faculty of Health Sciences, Jagiellonian University Medical College, Krakow, Poland

${ }^{2}$ Paediatric Division, Department of Thoracic Surgery, Institute for Tuberculosis and Lung Diseases, Rabka-Zdroj, Poland

A - Study Design, B - Data Collection, C - Statistical Analysis, D - Data Interpretation, E - Manuscript Preparation, F - Literature Search, G - Funds Collection

Summary Background. The prolonged survival of patients with cystic fibrosis leads to the search for predictors that determine their physical health and functioning in social and psycho-emotional areas, and allows for better understanding of the mutual interactions. Objectives. The aim of the study was to assess the incidence of pain in patients with CF and clinical determinants of anxiety, depression and aggression.

Material and methods. The study covered 95 patients with CF aged 14-25 years. The analysis included: the intensity of pain (Numeric Rating Scale), the level of anxiety, depression and aggression (Hospital Anxiety and Depression Scale), the quality of sleep (Athens Insomnia Scale), $\mathrm{FEV}_{1}$ and BMI.

Results. $42.1 \%(n=40)$ of patients with CF experienced pain, mainly moderate $(n=18 ; 45 \%)$ and severe $(n=14 ; 35 \%)$. In these patients, significantly higher median anxiety scores ( 6 vs 4 ) and median depression scores ( 3.5 vs 2 ) were recorded than in subjects without pain. Factors considerably determining anxiety scores were AIS scores $(\beta=0.56)$ and $\operatorname{sex}(\beta=0.22)$, while depression scores were determined by AIS scores $(\beta=0.56)$, BMI $(\beta=-0.20)$ and pain intensity $(\beta=0.20)$. In turn, sex $(\beta=0.30)$ and AIS scores $(\beta=0.19)$ had an influence on aggression scores.

Conclusions. Pain occurs in a large proportion of patients with CF. Factors determining emotional disorders in patients with CF are pain, female sex, quality of sleep and body weight. Anxiety, depression and aggression have always been accompanied by poorer sleep quality.

Key words: sleep, depression, pain, anxiety, cystic fibrosis, adolescent, adult.

Cepuch G, Tomaszek L, Pawlik L. Identification of factors determining anxiety, depression and aggression, with particular emphasis on pain in Polish adolescents and young adults with cystic fibrosis. Fam Med Prim Care Rev 2018; 20(2): 112-116, doi: https://doi. org/10.5114/fmpcr.2018.76452.

\section{Background}

Despite the fact that the development of medicine allows for better understanding and opens new pathways for the treatment of cystic fibrosis (CF), the condition continues to be a life-threatening disorder [1].

The prolonged survival of patients with CF leads to the search for predictors that determine their physical health and functioning in social and psycho-emotional areas, and allow for better understanding of the mutual interactions. The biological state of a patient with CF is already well understood and described [2], but the links between the biological state and other factors, for instance emotional, require further deepening of research.

Identified factors destabilizing the condition of a patient with CF include pain [3], sleep disorders [4] and negative emotions [5]. Pain is considered to be common in patients with CF, but its connotation and importance for the patient's functioning still appear to be underestimated. The pain problem in CF is sometimes ignored, and assumed to be an unspecific symptom. Clinical practice suggests that pain in this group of patients may accompany osteoporosis, spinal curvature, arthritis, sinusitis, emphysema and complications in the alimentary tract [6]. Also, physiotherapy may cause muscle tension that induces chest and back pain, and conversely, intense dyspnea and cough may cause painful contractions of intercorsal muscles [7]. Pain is a common problem in both children and adults with CF. It has negative clinical associations with life quality and the ability to successfully undertake treatment $[3,8]$.

Pain is also associated with depression, and depression may be a risk factor for the development of pain in patients with CF [9]. Negative emotions predispose patients towards higher perception of pain on one hand, but on the other hand, continuous pain, in its effects, may influence the incidence of negative emotions. Although epidemiological studies continue to provide data and information that could lead to a better understanding of the clinical manifestations of the disease, there are still many controversies regarding the importance of some risk factors, such as pain and depression in the pathogenesis of this disorder, and its management [10]. The findings emphasis the need to screen patients with CF for symptoms of depression and to treat co-morbid depression. There is an important need to continue to promote an integrated team approach to CF care, and to underline the need for multidisciplinary CF care teams to include a clinical psychologist.

Pain can lead to sleep disorders, which depend on the severity of the disease, the patient's sensitivity, previous treatments, 
and the efficacy of analgesics. The combination of symptoms caused by the disorder and sleep problems produce deeper physical and mental discomfort, leading to disorganization of social and individual functioning [11]. Assessing the mutual interactions between anxiety, depression and aggression, with particular emphasis on pain and quality of sleep, is necessary, especially in adolescents and young adults with CF. Young people may not have fully developed skills of coping with the disease and therapy, and developmental tasks may make them particularly susceptible to emotional stress. Anxiety seems to be the basic background for emotional life, against which anger, annoyance, depression, lack of self-confidence, helplessness and the loss of control over the body appear.

\section{Objectives}

The aim of the study was to assess the incidence of pain in patients with CF, and to examine the demographic and clinical determinants of anxiety, depression and aggression.

\section{Materials and methods}

\section{Setting}

The study comprised 95 subjects with CF, both sexes, aged 14-25 years, who reported to the follow up visit at the Institute of Tuberculosis and Lung Diseases in Rabka Zdroj.

The research was carried out between February 10, 2016 and February 28, 2017, and was approved by the Bioethics Commission of the Jagiellonian University in Krakow (No. 122.6120.275.2015).

\section{Participans}

The inclusion criterion was stable state of health in the 4 weeks prior to enrolment (no worsening of clinical status, e.g. aggravation of dyspnea or cough, coughing up sputum, the use of assisted breathing or respirotherapy). The study excluded patients who did not consent to participate in the study (the legal guardian's consent was necessary for patients under the age of 18), those after lung transplantation, or with mental or terminal diseases, and people who had traumatic experiences in the last 6 months (e.g. relationship breakdown, death of a significant person, job loss).

The level of pain intensity and pain relief were assessed using the Numeric Rating Scale, where 0 meant no pain/no pain relief, and 10 maximum pain/complete pain relief. The results of pain assessment were interpreted as: weak $(1-3 / 10)$, moderate (4-6/10), and strong (over 6/10).

The Hospital Anxiety and Depression Scale (HADS), which was modified to assess the level of aggression and validated to Polish conditions, was used to evaluate anxiety and depression. In depression and anxiety subscales, the patient can obtain from 0 to 21 points (normal: $0-7$ points, borderline: $8-10$ points, clinical levels of symptoms $\geq 11$ points); in the aggression subscale from 0 to 6 points (the higher the number of points, the higher the level of aggression).

The Polish version of the Athens Insomnia Scale (AIS) was used to assess the quality of sleep. If the symptom occurred at least three times a week during the last month, the patient assessed its severity on a scale of $1-3$ points ( 0 was no symptom). A total score of at least $8 / 24$ points may indicate insomnia.

A metric of our own design has been used to collect sociodemographic data and basic information about the patient's

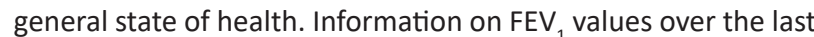
4 weeks was obtained from patients' medical records.

\section{Statistical methods}

Statistical analysis was performed using Statistica 12 software, including the "Medical Bundle" (StatSoft ${ }^{\circledR}$, Poland). Intergroup differences for quantitative variables were verified using the $t$-student or Mann-Whitney test based on the distribution of variables, while chi $^{2}$ and Fisher's exact test were used for qualitative variables. Correlations between values of numerical parameters were determined on the basis of Spearman's $(R)$ rank correlation coefficient.

A linear stepwise reverse regression was used to investigate demographic and clinical factors affecting anxiety, depression and aggression. The sum of points obtained in anxiety, depression and aggression subscales was a dependent variable. The logistic regression model was used to calculate the odds ratio for anxiety and depressive disorders depending on gender and the presence of insomnia. Statistical significance was assumed at $p=0.05$.

\section{Results}

\section{Participants and descriptive date}

Statistical analysis was based on the results from 95 questionnaires that were completed mostly by male patients $(56.8 \%)$, aged $17-25$ years $(73.7 \%)$. Patients who declared experiencing pain were assigned to the pain group $(42.1 \%)$, while those who did not feel pain were assigned to the no pain group (57.9\%). Demographic and clinical characteristics of patients are presented in Table 1.

\begin{tabular}{|c|c|c|c|c|}
\hline \multicolumn{2}{|l|}{ Parameter } & \multirow{2}{*}{$\begin{array}{l}\text { Pain } \\
(n=40) \\
9(22.5 \%)\end{array}$} & \multirow{2}{*}{\begin{tabular}{|l|}
$\begin{array}{l}\text { No pain } \\
(n=55)\end{array}$ \\
$16(29.1 \%)$ \\
\end{tabular}} & \multirow{3}{*}{\begin{tabular}{|l}
$p$ \\
$\chi^{2}=0.577 ;$ \\
$p=0.749$
\end{tabular}} \\
\hline \multirow[t]{3}{*}{ Age (years) } & $14-16$ & & & \\
\hline & $17-20$ & $15(37.5 \%)$ & $20(36.4 \%)$ & \\
\hline & $21-25$ & $16(40.0 \%)$ & 19 (34.5\%) & \\
\hline \multirow[t]{2}{*}{ Sex } & Girls & $20(50.0 \%)$ & $21(38.2 \%)$ & \multirow{2}{*}{$\begin{array}{l}\chi^{2}=1.318 \\
p=0.250\end{array}$} \\
\hline & Boys & $20(50.0 \%)$ & $34(61.8 \%)$ & \\
\hline \multicolumn{2}{|l|}{$\mathrm{BMI}\left(\mathrm{kg} / \mathrm{m}^{2}\right)$} & $19.6 \pm 2.5$ & $20.1 \pm 3.1$ & $\begin{array}{l}t=-0.706 \\
p=0.481\end{array}$ \\
\hline \multicolumn{2}{|c|}{$\mathrm{FEV}_{1}(\%$ of pred.) } & $68.6 \pm 25.4$ & $67.9 \pm 20.7$ & $\begin{array}{l}t=-0.144 ; \\
p=0.885\end{array}$ \\
\hline \multicolumn{2}{|c|}{ Anxiety (scores) } & $6(4-10)$ & $4(2-8)$ & $\begin{array}{l}Z=-1.994 ; \\
p=0.046\end{array}$ \\
\hline \multicolumn{2}{|c|}{ Depression (scores) } & $3.5(2-7.5)$ & $2(1-6)$ & $\begin{array}{l}Z=-2.199 \\
p=0.027\end{array}$ \\
\hline \multicolumn{2}{|c|}{ Aggression (scores) } & $3.5(2-4)$ & $3(2-4)$ & $\begin{array}{l}Z=-0.893 ; \\
p=0.371\end{array}$ \\
\hline \multicolumn{2}{|l|}{ AIS (scores) } & $6.5(4-10)$ & $5(3-8)$ & $\begin{array}{l}Z=-1.239 ; \\
p=0.214\end{array}$ \\
\hline \multirow{2}{*}{$\begin{array}{l}\text { Co-morbidi- } \\
\text { ties* }\end{array}$} & yes & $16(40.0 \%)$ & $29(52.7 \%)$ & \multirow{2}{*}{$\begin{array}{l}\chi^{2}=1.504 \\
p=0.219\end{array}$} \\
\hline & no & $24(60.0 \%)$ & $26(47.3 \%)$ & \\
\hline
\end{tabular}

Results presented as medians (interquartile ranges), mean (standard deviation) or numbers (percentages). Abbreviations: BMI - body mass index; $\mathrm{FEV}_{1}$ - forced expiratory volume in $1 \mathrm{~s}$; AIS - Athens Insomnia Scale, $*$ the co-morbidities included diabetes $(n=16)$, bronchial asthma $(n=15)$, nasal polyps $(n=10)$ and cholelithiasis $(n=8)$.

\section{Main results}

\section{Prevalence of pain}

In the pain group 8 patients (20\%) suffered from mild, 18 from moderate $(45 \%)$ and 14 from strong pain $(35 \%)$. 
The most common was pain of the abdomen (50\%), head $(47.5 \%)$ and chest $(37.5 \%)$. Less frequently, pain was felt in the muscles (12.5\%), spine (5\%) and joints (5\%). One source of pain was found in 35 (87.5\%) patients, while in the remaining subjects it was located in several areas of the body. There was a moderate correlation between pain intensity and the number of pain sources $(R=0.44 ; t=3.081 ; p=0.003)$.

Analgesic treatment was mainly based on the immediate administration of non-steroidal anti-inflammatory drugs and/ /or paracetamol and/or metamizol (70\%). Patients took medications mainly after physical activity, when the pain intensity was $\geq 3 / 10$ (mean pain relief after analgesic treatment was $7.7 \pm 1.8$ ).

\section{The evaluation of the relationship between pain, emotional disorders and the quality of sleep}

Patients with pain had significantly higher median anxiety scores and median depression scores than those without pain (Table 1). Pain intensity poorly correlates with anxiety scores $(R=0.25 ; t=2.498 ; p=0.014)$ and depression scores $(R=0.27$; $t=2.277 ; p=0.006$ ).

There was no significant difference between patients from the pain and no pain groups in terms of aggression scores and AIS scores (Table 1 ).

\section{The evaluation of emotional disorder determinants}

\section{Anxiety}

In the whole study group, anxiety disorders were diagnosed in 16 subjects (16.8\%) and "borderline cases" in 17 (17.9\%) patients with CF.

Factors determining anxiety scores were AIS scores and sex. This model explained $41 \%$ of anxiety scores variable variances (Table 2, model 1). If an insomnia variable was introduced into the regression model instead of AIS scores, then the model explained $31 \%$ of anxiety scores variable variances (Table 2 , model 2 ).

\begin{tabular}{|c|c|c|c|c|c|c|}
\hline Predictors & $\beta$ & $\begin{array}{l}\text { SE } \\
\text { of } \beta\end{array}$ & $b$ & $\begin{array}{l}\text { SE } \\
\text { of } b\end{array}$ & $t$ & $p$ \\
\hline \multicolumn{7}{|c|}{ Model 1. $R^{2}=0.41 ; F(2.92)=33.040 ; p=0.00$} \\
\hline AIS scores & 0.56 & 0.08 & 0.49 & 0.07 & 6.935 & 0.000 \\
\hline Sex Women & 0.22 & 0.08 & 0.95 & 0.34 & 2.758 & 0.007 \\
\hline \multicolumn{7}{|c|}{ Model 2. $R^{2}=0.31 ; F(2.92)=21.635 ; p=0.00$} \\
\hline Insomnia & 0.45 & 0.08 & 1.99 & 0.37 & 5.280 & 0.000 \\
\hline Sex Women & 0.26 & 0.08 & 1.13 & 0.36 & 3.075 & 0.002 \\
\hline
\end{tabular}

$\beta$-standardized regression coefficient; $b$ - non-standardized regression coefficient; SE - standard error; $R^{2}$-determination coefficient; AIS - Athens Insomnia Scale.

Median anxiety scores were significantly higher in patients with insomnia than in those with good quality of sleep (10 vs $4 ; Z=-4.549 ; p=0.000$ ), and in women than in men ( 7 vs 4 , $Z=3.354 ; p=0.000$ ). The odds of anxiety disorders are 5.2 times higher in female patients with CF than in male patients (OR: 5.17; $95 \% \mathrm{Cl}: 1.52$ to 17.53 ).

\section{Depression}

Depression was diagnosed in 6 patients $(6.3 \%)$, and there were 12 "borderline cases" (12.6\%) among subjects with CF. Depression scores strongly correlated with anxiety scores $(R=0.72 ; t=10.005 ; p=0.000)$. Factors determining depression scores were: AIS scores, BMI and pain intensity. These variables explained $43 \%$ of depression scores variable variances (Table 3).

\begin{tabular}{|c|c|c|c|c|c|c|}
\hline Predictors & $\beta$ & $\begin{array}{l}S E \\
\text { of } \beta\end{array}$ & $b$ & $\begin{array}{l}\text { SE } \\
\text { of } b\end{array}$ & $t$ & $p$ \\
\hline AIS scores & 0.56 & \begin{tabular}{|l|}
0.08 \\
\end{tabular} & 0.42 & 0.061 & 6.933 & 0.000 \\
\hline BMI & -0.20 & 0.07 & -0.27 & 0.104 & -2.619 & 0.010 \\
\hline Pain intensity & 0.20 & 0.08 & 0.23 & 0.097 & 2.435 & 0.016 \\
\hline
\end{tabular}

$\beta$ - standardized regression coefficient; $b$ - non-standardized regression coefficient; SE - standard error; $R^{2}$ - determination coefficient; AIS - Athens Insomnia Scale; $R^{2}=0.43 ; F(3.91)=22.560 ; p=0.000$.

\section{Aggression}

Aggression scores were determined by sex and AIS scores. Gender and AIS scores explained $15 \%$ of aggression variable variances (Table 4$)$. The level of aggression moderately correlated with the level of anxiety $(R=0.41 ; t=4.401 ; p=0.000)$. Significantly higher levels of aggression were recorded in women than in men (Me: 4 vs $2.5 ; Z=3.249 ; p=0.000$ ). The odds of a higher level of aggression in female patients with CF are 1.6 times greater than in male patients (OR: $1.6 ; 95 \% \mathrm{Cl}: 1.19$ to 2.14).

\begin{tabular}{|c|c|c|c|c|c|c|}
\hline Predictors & $\beta$ & SE of $\beta$ & $b$ & SE of $b$ & $t$ & $p$ \\
\hline Sex Women & 0.30 & 0.09 & 0.47 & 0.15 & 3.066 & 0.002 \\
\hline AIS scores & 0.19 & 0.09 & 0.06 & 0.03 & 1.994 & 0.049 \\
\hline
\end{tabular}

$\beta$-standardized regression coefficient; $b$ - non-standardized regression coefficient; SE - standard error; $R^{2}$ - determination coefficient; AIS - Athens Insomnia Scale; $R^{2}=0.15 ; F(2.92)=8.254 ; p=0.000$.

\section{Discussion}

\section{Key results}

Our study revealed that a significant proportion of CF adolescents and young adults experienced pain, as in the study conducted by Festini et al. [6]. Nearly $90 \%$ of the subjects indicated one source of pain, among which the digestive tract, head and respiratory system dominated. Lechnitz et al. [8] and Hayes et al. [12] obtained similar results in terms of pain location, whereas in the study conducted by Moraru et al. [10] nearly $34 \%$ of patients complained of various pain manifestations.

The study demonstrated a positive correlation between the level of pain intensity and the number of its source, but the correlation was moderate. Analgesia used by patients was primarily based on the immediate oral administration of paracetamol, metamizol and non-steroidal anti-inflammatory drugs. In the subjects' opinion, analgesic treatment brought considerable pain relief. The results could have been influenced by the stable state of the patient's health, but the effectiveness of analgesia could also result from good education about pain management by the medical team, especially nurses. Given the amount of time devoted to patients, nursing teams can use their professional competence to provide patients with information support for pharmacological and non-pharmacological methods of fighting pain and reducing anxiety [13].

The analysis of the material has confirmed the relationship between pain and higher levels of anxiety and depression. Similar results were obtained by Hayes et al. [12], proving that symptoms of depression and anxiety were more common among patients with pain. Importantly, the authors have demonstrated the association of pain not only with the greater risk of negative emotional states, but also with the higher probability of death.

The relationship between pain and aggression was not shown in our study. Higher levels of aggression have been re- 
ported in female patients and those with sleep disorders. Higher levels of aggression also correlated with greater anxiety. It is difficult to compare the results because it was not possible to find studies based on the HADS scale extended by the part diagnosing aggression.

The problem of anxiety and depression is of particular importance among patients with CF due to the connotation with the patient's biological state. Being subjective experiences, anxiety and depression coexist with neurophysiological changes, and thus they can also somatise [14]. The studied emotions are also capable of mutual interactions, and by worsening the condition of a patient with CF they become determinants of his/ /her survival time $[15,16]$. Duff et al. [17] demonstrated that feeling unwell was associated with increased depression scores, whereas Dantzer et al. [18] highlights the association between pathophysiological processes, such as inflammation, and increased depressive symptoms identified in the context of other medical conditions. The relationship between depression and anxiety was confirmed in our study results. The severity of anxiety and depressive symptoms was dependent on the quality of sleep. Matsuda and Tully [19] reported a relationship between poor quality of sleep and increased risk of depression and anxiety. In turn, Backström-Eriksson et al. [20] noted that females are more likely to suffer from anxiety disorders, as confirmed by our results.

Apart from the quality of sleep and anxiety, the level of depression was also affected by low BMI and pain intensity. There have been no reports on the association between $\mathrm{BMI}$ and depression in patients with CF. The literature mainly contains reports on the relationship between depression and overweight/ /obesity or anorexia [21, 22]. In patients with cystic fibrosis, $\mathrm{BMI}$ is a measure of physical well-being and good health control. Thus, a decrease in BMI can be perceived by the patient as a sign of deterioration of the health condition and, consequently, a shortening of survival time, becoming a factor destabilizing the psycho-emotional state.

The results of our study clearly indicate the relationship between pain, weight and sleep disorders, including insomnia, and the development of negative emotional states. This is of importance both for the patient and therapeutic teams and institutions providing medical care. Mutual connotations between the study variables hinder the clinical assessment of the patient's condition and generate considerable costs for the treatment of these patients. At the same time, making a diagnosis of some negative emotional states can be difficult because of the wide range of symptoms, especially in young people [23]. Understanding the interactions between these variables is very important in the treatment process $[24,25]$ (both for nurses, physicians and patients).

\section{Why are the study results important?}

The research tools are easy to use and can be applied by nursing teams and physicians. They serve for the examination of both young and adult patients, enabling us to objectify the results in different age groups. The examination of patients with risk factors, such as female gender, sleep disorders and pain is particularly indicated.

\section{The implications for clinical practice}

The popularization of the need for screening in the study area can improve the well-being of patients and reduce the cost of treatment.

\section{Limitations of the study}

The evaluation of anxiety and depression using only a screening tool, sleep assessment without objective tests (e.g. polysomnography), no control group, the lack of information on how often the subjects reported pain during the day, and the distinction between acute and chronic pain.

\section{Conclusions}

Factors determining emotional disorders in patients with CF include female gender, the quality of sleep, pain intensity and BMI. Anxiety, depression and aggression are always accompanied by poor quality of sleep. The greater the pain intensity and the lower the BMI, the higher the severity of depressive symptoms in patients with CF.

Source of funding: This work was supported in part by Grant No. 10.10 from the National Tuberculosis and Lung Diseases Research Institute, Poland.

Conflicts of interest: The authors declare no conflicts of interest.

\section{References}

1. Krvavac A, Nayak RP. New horizons in cystic fibrosis - a review. JSM Gastroenterol Hepatol 2016; 4(2): 2-9.

2. Prandini P, De Logu F, Fusi C, et al. Transient receptor potential ankyrin 1 channels modulate inflammatory response in respiratory cells from patients with cystic fibrosis. Am J Respir Cell Mol Biol 2016; 55(5): 645-656.

3. Lee AL, Rawlings S, Bennett KA, et al. Pain and its clinical associations in individuals with cystic fibrosis: a systematic review. Chron Respir Dis 2016; 13(2): 102-117.

4. Perin C, Fagondes S, Casarotto F, et al. Sleep findings and predictors of sleep desaturation in adult cystic fibrosis patients. Sleep Breath 2012; 16(4): 1041-1048.

5. Baiardini I, Steinhilber G, DI Marco F, et al. Anxiety and depression in cystic fibrosis. Minerva Med 2015; 106(5 Suppl. 1): 1-8.

6. Festini F, Ballarin S, Codamo T, et al. Prevalence of pain in adults with cystic fibrosis. J Cyst Fibros 2004; 3: 51-57.

7. Sermet-Gaudelus I, De Villartay P, de Dreuzy P, et al. Pain in children and adults with cystic fibrosis: a comparative study. $J$ Pain Symptom Manage 2009; 38(2): 281-290.

8. Lechnitz N, Allgood S, Hong G, et al. The association between pain and clinical outcomes in adolescents with cystic fibrosis. J Pain Symptom Manage 2016; 52(5): 681-687.

9. Hog G, Allgood S, Riekert KA, et al. The association between pain and depression in adolescents with cystic fibrosis. Am J Respir Crit Care Med 2014; 189: A4032.

10. Moraru E, Diaconu R, Anton E, et al. Understanding the relevance of pain, depression and anxiety - like manifestations in the management of cystic fibrosis. Arch Biol Sci 2015; 67(3): 10671-10070.

11. Annagür BB, Uguz F, Apiliogullari S, et al. Psychiatric disorders and association with quality of sleep and quality of life in patients with chronic pain: a SCID-based study. Pain Med 2014; 15: 772-781.

12. Hayes $\mathrm{M}$, Yaster $\mathrm{M}$, Haythornthwaite $\mathrm{JA}$, et al. Pain is a common problem affecting clinical outcomes in adults with cystic fibrosis. Chest 2011; 140(6): 1598-1603.

13. Fortier MA, Kain ZN. Treating perioperative anxiety and pain in children: a tailored and innovative approach. Paediatr Anaesth 2015; 25(1): 27-35. 
14. Lisboa SF, Gomes FV, Terzian AL, et al. The endocannabinoid system and anxiety. Vitam Horm 2017; 103: 193-279.

15. Pratt LA, Druss BG, Manderscheid RW, et al. Excess mortality due to depression and anxiety in the United States: results from a nationally representative survey. Gen Hosp Psychiatry 2016; 39: 39-45.

16. Fidica A, Herle M, Goldbeck L. Symptoms of depression impact the course of lung function in adolescents and adults with cystic fibrosis. BMC Pulm Med 2014; 14: 205.

17. Duff AJ, Abbott J, Cowperthwaite $C$, et al. Depression and anxiety in adolescents and adults with cystic fibrosis in the UK: a crosssectional study. J Cyst Fibros 2014; 13(6): 745-753.

18. Dantzer R, O'Connor JC, Freund GG, et al. From inflammation to sickness and depression: when the immune system subjugates the brain. Nat Rev Neurosci 2008; 9: 46-56.

19. Matsuda A, Tully EC. The role of mindfulness and psychological flexibility in somatization, depression, anxiety, and general psychological distress of a non-clinical college sample. J Evid-Based Complement Alternat Med 2012; 17(1): 66-71.

20. Backström-Eriksson L, Sorjonen K, Bergsten-Brucefors A, et al. Anxiety and depression in adults with cystic fibrosis: a comparison between patients and the general population in Sweden and three other European countries. BMC Pulm Med 2015; 15(1): 1-7.

21. Gaysina D, Hotopf M, Richards $M$, et al. Symptoms of depression and anxiety, and change in body mass index from adolescence to adulthood: results from a British birth cohort. Psychol Med 2011; 41(1): 175-184.

22. Sidor A, Baba CO, Marton-Vasarhelyi E, et al. Gender differences in the magnitude of the associations between eating disorders symptoms and depression and anxiety symptoms. Results from a community sample of adolescents. J Ment Health 2015; 24 (5): $294-298$.

23. Carrellas NW, Biederman J, Uchida M. How prevalent and morbid are subthreshold manifestations of major depression in adolescents? A literature review. J Affect Disord 2017; 210: 166-173.

24. Nowicka-Sauer K, Pietrzykowska M, Staśkiewicz I, et al. Anxiety in patients with somatic diseases: important but marginalized problem. Fam Med Prim Care Rev 2015; 17(2): 120-123.

25. Quittner AL, Saez-Flores E, Barton JD. The psychological burden of cystic fibrosis. Curr Opin Pulm Med 2016; 22(2): 187-191.

Tables: 4

Figures: 0

References: 25

Received: 13.09 .2017

Reviewed: 19.09 .2017

Accepted: 01.11.2017

Address for correspondence:

Grażyna Cepuch, PhD

Zakład Pielęgniarstwa Klinicznego

Instytut Pielęgniarstwa i Położnictwa UJ CM

ul. Kopernika 25

31-501 Kraków

Polska

Tel.: +48 600 132-585

E-mail: grazyna.cepuch@uj.edu.pl 